\title{
Diagnostics of Particles emitted from a Laser generated Plasma: Experimental Data and Simulations
}

\author{
Giuseppe Costa ${ }^{1, *}$ and Lorenzo Torrisi ${ }^{1}$ \\ ${ }^{1}$ Dipartimento di Scienze Fisiche, MIFT, Università di Messina, V.le F.S. D’Alcontres31, 98166 S. Agata, Messina, Italy
}

\begin{abstract}
The charge particle emission form laser-generated plasma was studied experimentally and theoretically using the COMSOL simulation code. The particle acceleration was investigated using two lasers at two different regimes. A Nd:YAG laser, with $3 \mathrm{~ns}$ pulse duration and $10^{10} \mathrm{~W} / \mathrm{cm}^{2}$ intensity, when focused on solid target produces a non-equilibrium plasma with average temperature of about 30-50 eV. An Iodine laser with $300 \mathrm{ps}$ pulse duration and $10^{16} \mathrm{~W} / \mathrm{cm}^{2}$ intensity produces plasmas with average temperatures of the order of tens $\mathrm{keV}$. In both cases charge separation occurs and ions and electrons are accelerated at energies of the order of $200 \mathrm{eV}$ and $1 \mathrm{MeV}$ per charge state in the two cases, respectively. The simulation program permits to plot the charge particle trajectories from plasma source in vacuum indicating how they can be deflected by magnetic and electrical fields. The simulation code can be employed to realize suitable permanent magnets and solenoids to deflect ions toward a secondary target or detectors, to focalize ions and electrons, to realize electron traps able to provide significant ion acceleration and to realize efficient spectrometers. In particular it was applied to the study two Thomson parabola spectrometers able to detect ions at low and at high laser intensities. The comparisons between measurements and simulation is presented and discussed.
\end{abstract}

\section{Introduction}

Laser Generated Plasma (LGP) in vacuum is a relevant topic in many scientific fields, due to its large number of applications, such as preparation of ion sources, ion acceleration method, production of nuclear reactions, generation of neutron emission $[1,2]$.

In many applications ion beams with adequate characteristics are required, including energy and charge state distributions, emittance, high electron and ion currents.

Magnetic fields can be applied to LGPs in order to focus the charged particles emitted from the irradiated target or to select particles with definited energy using longitudinal or transversal magnetic fields, respectively. These two cases are performed at the Plasma Physics Laboratory in Messina University, using permanent magnetic fields with maximum values of about $0.3 \mathrm{~T}$. A simulation software called "COMSOL Multiphysics" [3] was employed in this study, and to compare model results with experimental data.

When electric fields were associated to magnetic fields, they allow to get a better resolution for the mass-tocharge ratio measurements and for the energy measure of the particles emitted from the target.

Magnetic fields can be also employed to realize electron traps acting on the flux and on the energy of the plasma accelerated charged particles, according to the literature [4].
Electro-magnetic (EM) fields are used to realize the appropriate spectrometers known as the "Thomson Parabola Spectrometer" (TPS) [5].

In this paper, we focus on particles emitted from targets irradiated by low and medium laser intensity, i.e. from $10^{10}$ up to $10^{16} \mathrm{~W} / \mathrm{cm}^{2}$ intensities, respectively.

\section{Materials and methods}

In this work we have considered three systems that use magnetic and electrical fields to study a non-equilibrium plasma generated by laser pulses:

1. Longitudinal Magnetic Field: consisting of a magnetic field having a symmetry axis parallel to the main plasma plume emission, and hence orthogonal to the irradiated target.

2. Transverse Magnetic Field: consisting of a magnetic field having lines orthogonal to the main propagation direction of the nonequilibrium plasma, and hence parallel to the target surface.

3. Transverse Magnetic and Electrical Field: called Thomson Parabola Spectrometer (TPS), consisting of a magnetic and an electrical field transverse to the main propagation direction of the plasma, i.e. parallel to the target surface.

\footnotetext{
Corresponding author: gcosta@unime.it
} 


\subsection{Longitudinal Magnetic Field}

A Nd:YAg laser, with fundamental wavelength 1064 $\mathrm{nm}$, maximum pulse duration $3 \mathrm{~ns}$, and energy variable from 1 to $300 \mathrm{~mJ}$, was employed to irradiate dielectric and metallic targets, in single shot mode. The laser beam is focused on the target, placed in a vacuum chamber at a pressure of about $10^{-6}$ mbar, through an optical lens having a focal distance of $50 \mathrm{~cm}$. The spot area on the target surface is about $0.5 \mathrm{~mm}^{2}$. The laser beam incidence angle on the target surface is $45^{\circ}$. The irradiated targets are constituted by polyethylene $\left(-\mathrm{CH}_{2}\right.$ monomer) and pure aluminium. A scheme and picture of the experimental setup are reported in Figure 1a and 1b, respectively [6].
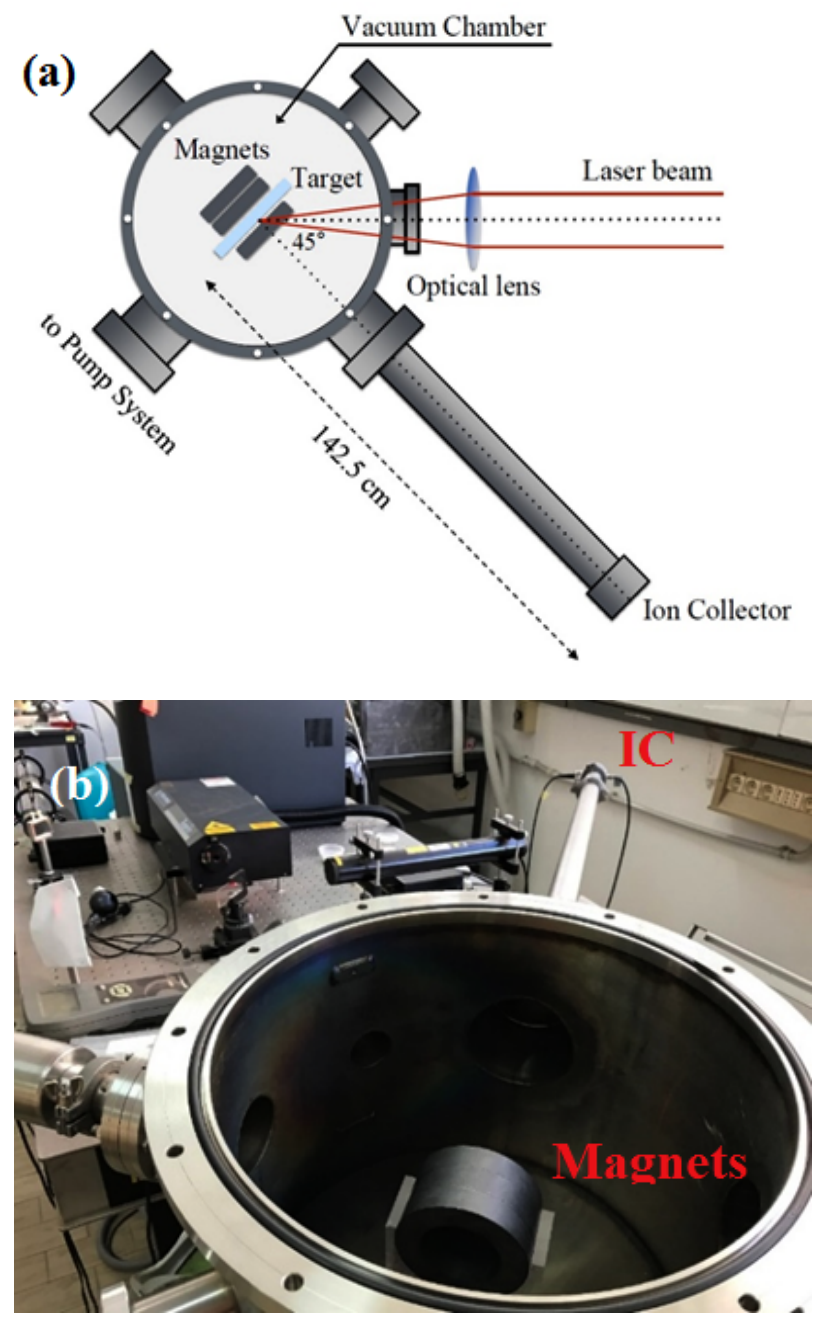

Fig. 1. Scheme (a), and picture (b) of the experimental setup for use of Longitudinal Magnetic field.

The magnet rings are permanent ferrite magnets. A single magnet has an outer diameter of $100 \mathrm{~mm}$ and an internal of $60 \mathrm{~mm}$; its thickness is $20 \mathrm{~mm}$. The produced magnetic field is axial $(0.12 \mathrm{~T}$ on the surface of the magnet and $0.035 \mathrm{~T}$ at the centre of it). The axis of the magnets is placed along the normal to the target surface, in correspondence of the laser spot position.

The experiment was conducted without magnets, and with one circular magnet placed in front of the target and with one, two or four magnets placed behind the target.
The magnetic field module at the centre of magnets, accurately measured with a suitable Gaussmeter (Hirst GM08), ranges from $0.071 \mathrm{~T}$ to $0.12 \mathrm{~T}$.

The detector used to collect the ions emitted from the plasma, is an ion collector (IC), placed at $142.5 \mathrm{~cm}$ distance from the target. The IC is polarized to $-50 \mathrm{~V}$ with respect to the grid in order to reduce the secondary electron emission. This device is used in time of flight technique (TOF), which consists to evaluate the time that ions take to travel a known distance from the target to the detector, measured using a fast storage oscilloscope, as reported previously [6].

\subsection{Transverse Magnetic Field}

With the same laser as for polyethylene we irradiated a pure aluminium target. The particles emitted from the target cross a collimation system consisting of two pinholes with a smaller radius of $1.5 \mathrm{~mm}$. The collimated particle beams pass through the centre of a system of two permanent magnets. The magnetic field module is about $0.38 \mathrm{~T}$ and it has a length of $10 \mathrm{~mm}$, as shown in the plot of Fig. 2b. A group of 32 aligned Faraday Cups distributed at $90^{\circ}$ angle, and placed on a movable chest, as shown in Fig. 2a, reveal the deflected particles crossing the permanent magnet.

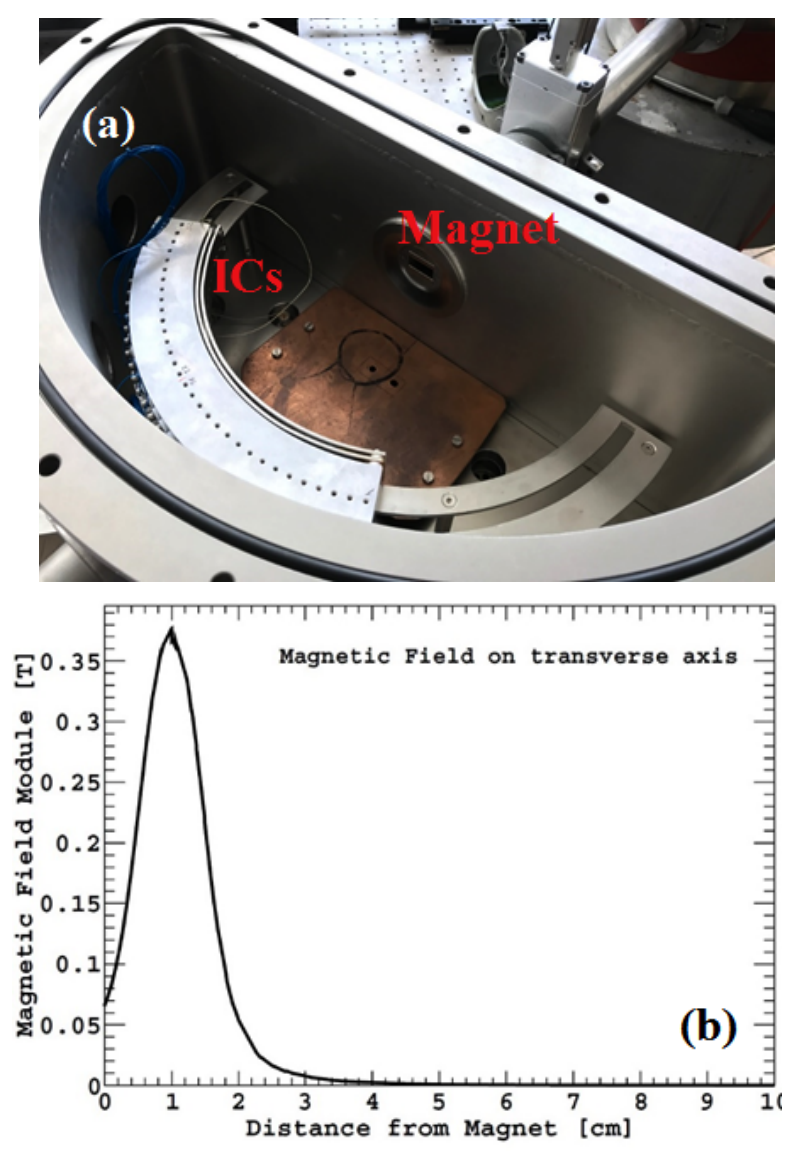

Fig. 2. Picture of the experimental setup (a). Magnetic Field Module, at the centre of the system (b). 


\subsection{Transverse Magnetic and Electrical Field}

In this case, two types of lasers are considered to produce a non-equilibrium plasma in high vacuum. In the first case, a Nd: YAG laser with $10^{12} \mathrm{~W} / \mathrm{cm}^{2}$ intensity, is used to irradiate a target of titanium in vacuum. The produced plasma is accelerated with a longitudinal post-acceleration system up to $30 \mathrm{keV}$ per charge state, as reported previously [7]. The postaccelerated ion beams go through a magnetic and electrical fields orthogonal to their propagation direction, relative to the TPS system, which separates them according to their charge-to-mass ratio, projecting the ions on an orthogonal shield on which they produce parabolic curves. The used TPS scheme is shown in Fig. $3 \mathrm{a}$. The TPS consists of two pinholes with diameters of 1 $\mathrm{mm}$ and $1 \mu \mathrm{m}$ respectively, which have the role to make the beam tight and collimated. The ions then pass through a transverse magnetic field with module of $0.2 \mathrm{~T}$ and a transverse electric field with module $1 \mathrm{kV} / \mathrm{cm}$, each $2.5 \mathrm{~cm}$ long, as shown in Fig. $3 \mathrm{~b}$. The ions travel 15 $\mathrm{cm}$ before being detected by a MCP (MicroChannel Plate) with phosphor screen. Finally the parabola are recorded through a glass windows with a fast CCD camera placed externally to the vacuum chamber.

This study was carried out at the National South Laboratories-INFN (LNS) in Catania [8].
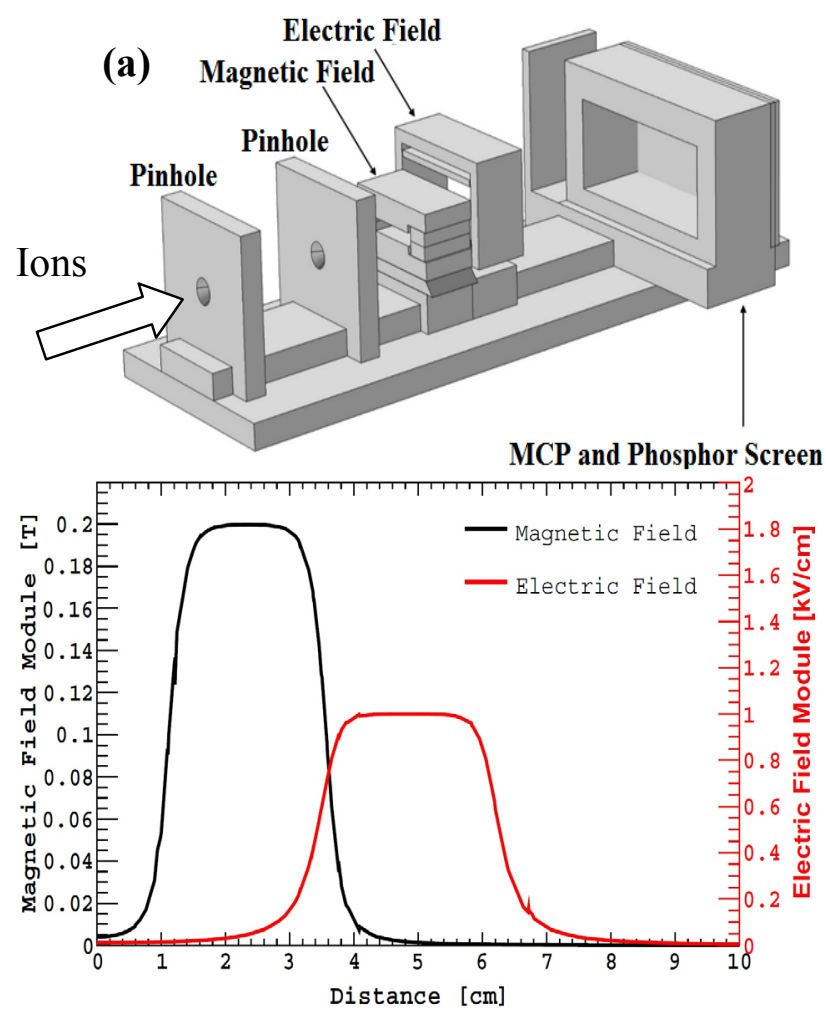

Fig. 3. Schematic (a), and magnetic and electric field module (b) applied at TPS of LNS (Catania).

In the second case, an Iodine laser with $10^{16} \mathrm{~W} / \mathrm{cm}^{2}$ intensity is used to irradiate a target of deuterated polyethylene. The laser beam forms a $0^{\circ}$ angle with the target, and the interaction regime is TNSA (Target Normal Sheath Acceleration) [9]. The plasma generated by laser accelerates charge particles towards a system of two pinholes, used as collimators and accurate alignment of the incident ion direction. The collimated ions cross a magnetic field with module of $0.1 \mathrm{~T}$ and an electrical field with module of $2 \mathrm{kV} / \mathrm{cm}$, each $8 \mathrm{~cm}$ long, as shown in Fig. 4b. The deflected particles travel $16.5 \mathrm{~cm}$ before impacting on a MCP with phosphor screen, as shown in Fig. 4a. This study was carried out at Prague Asterix Laser System (PALS), in Czech Republic [10].
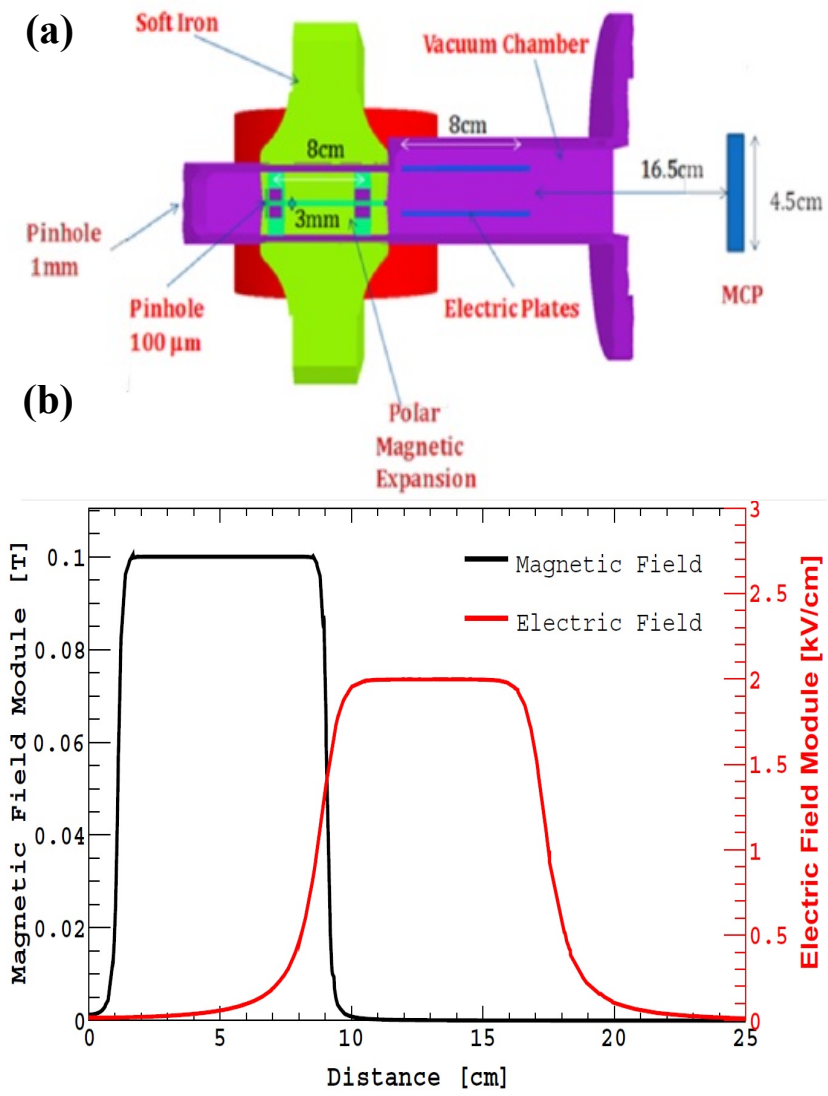

Fig. 4. Schematic (a), and magnetic and electrical field module (b) applied at TPS of PALS (Prague).

\section{Result and Discussion}

The main results obtained for the three experimental disposition above described are presented and discussed in the following.

\subsection{Longitudinal Magnetic Field}

In Figure 5 are shown the IC-TOF spectra obtained by irradiating targets of aluminium and glassy carbon, with and without the use of longitudinal magnetic fields.

Measurements demonstrate that the system of axial magnetic fields focuses the plasma ions enhancing the ion yield collected with the axial IC detector. The measurements of the ion current (yield) demonstrate that it increases when the axial magnetic field is applied by about a factor of 3.5 for aluminium target and by a factor of 2.5 for the glassy carbon target. Also experiments with the polyethylene target demonstrate an yield enhancement similar to that observed for carbon. 

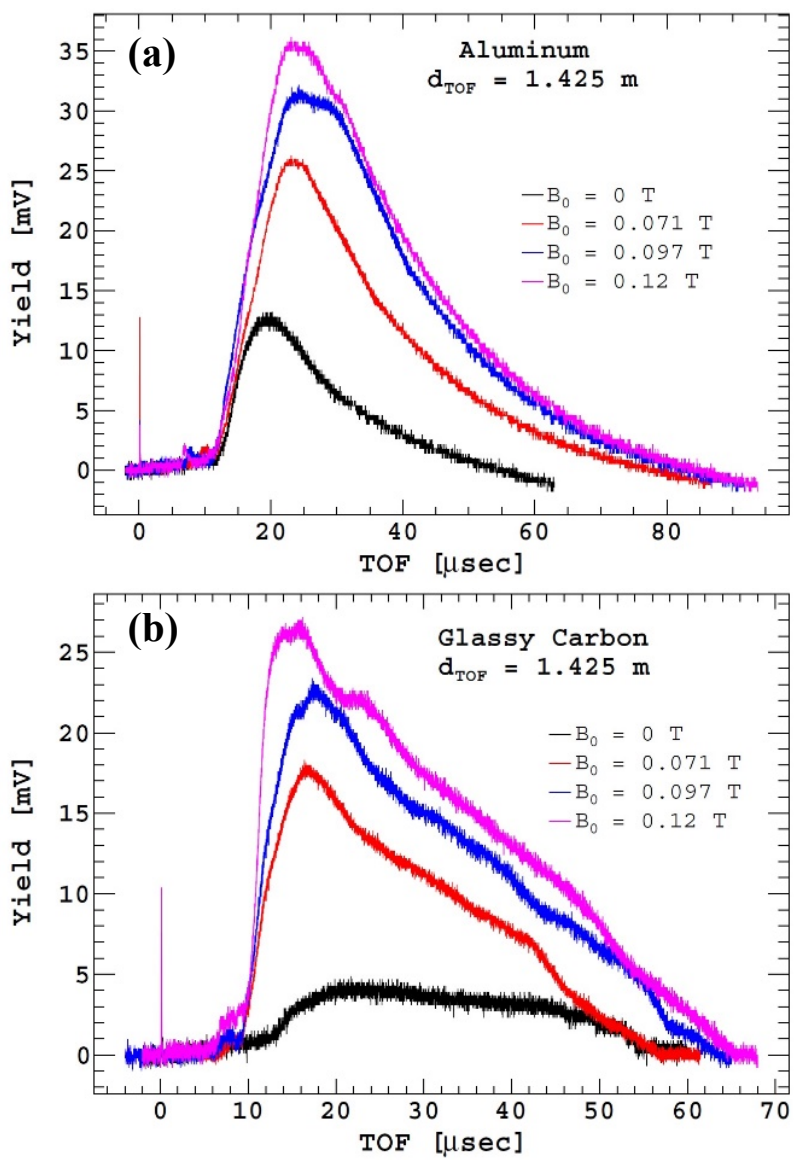

Fig. 5. Experimental data for pure Aluminium (a) and Glassy Carbon (b).
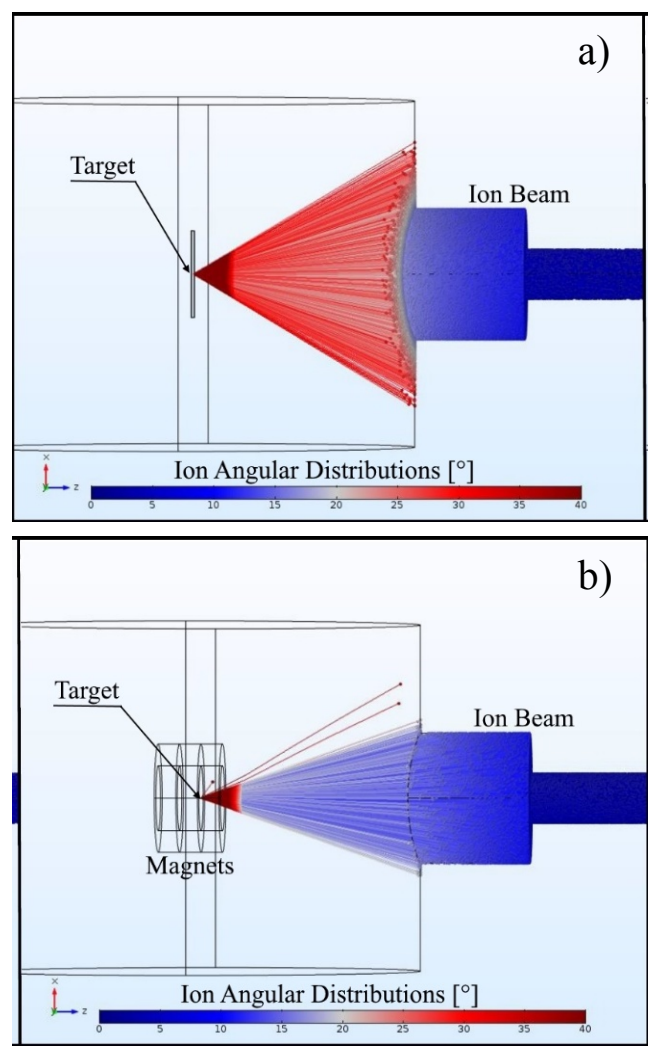

Fig. 6. Simulation with COMSOL for ion beam in the system without (a) and with (b) Magnets.
The increase of the ion yield was evaluated not only experimentally but also theoretically through the COMSOL-Multiphysics simulation program. An example is reported in Fig. 6 [6].

The simulation data have shown an excellent agreement with the experimental ones. In particular, the obtained experimental data and simulations show an increase in ion energy and ion yield when the axial magnetic field is applied. The energy increment for $\mathrm{H}^{+}$ions measured from the TOF spectra of Fig. 5 is of about $92 \%$ for carbon target and about $99 \%$ for aluminium target.

To explain the increase of measured ion energy it is necessary to know the dynamics of the plasma produced electrons in the system of interest. With the simulation software COMSOL it is possible to simulate the motion of the electrons in the magnetic fields with axial symmetry, as reported in Figure 7 [6].

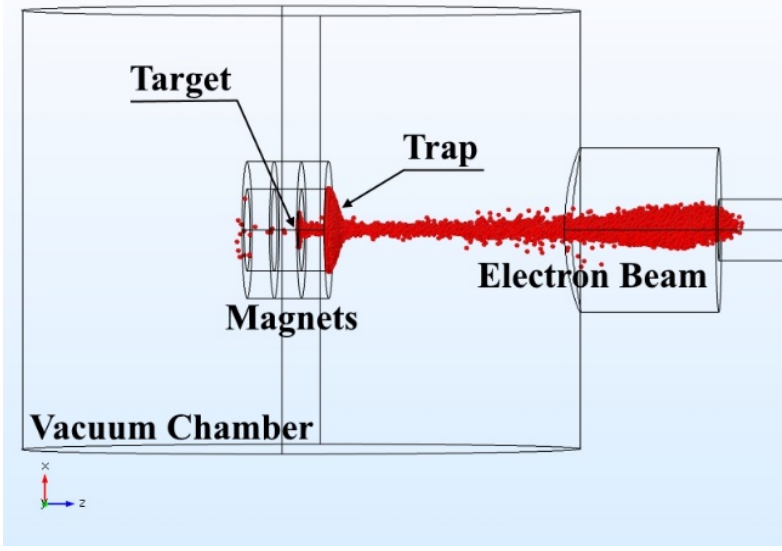

Fig. 7. Simulation with COMSOL for electron beam in the system with longitudinal Magnets.

In presence of the magnets, the kinetic electrons produce two traps, they form two negative clouds placed in front of the target surface that are responsible for an increase of the plasma potential and of the consequent ion acceleration. The perturbation of the emitted electron beams, due to the axial magnetic field, is responsible for the increase of plasma potential and consequently of the increase of the ion energy. The ion energy increases as a result of the electron density of the irradiated target and of the electron traps generated in front of the target surface [4].

\subsection{Transverse Magnetic Field}

In Fig. 8 are shown the highest ion yields displayed vs. the detection angle for a pure aluminium target. Since the energy distribution for the particles emitted by the target is the Coulomb-Boltzmann shifted [11]:

$$
f(E)=\frac{A}{\sqrt{2 m}} \sqrt{\frac{1}{\left(\pi k_{B} T\right)^{2}}} \operatorname{Eexp}\left[-\frac{1}{k_{B} T}\left(\sqrt{E}-\sqrt{\frac{\gamma k_{B} T}{2}}-\sqrt{z e V_{0}}\right)^{2}\right]
$$

where $\mathrm{A}$ is a normalization constant, $m$ is the mass of the ion considered, $\gamma$ represents the adiabatic expansion 
coefficient, $z e$ is the ion charge state, $\mathrm{V}_{0}$ is the equivalent potential in plasma, $\mathrm{k}_{\mathrm{B}}$ is the Boltzmann constant, and finally $\mathrm{k}_{\mathrm{B}} \mathrm{T}$ is the equivalent plasma temperature in $\mathrm{eV}$, the TOF spectra obtained using the spectrometer shown in Fig. 2a were investigated. Again, using the COMSOL simulation program, and implementing energy particle distribution of eq. (1), a comparison between experimental data and simulation can be done, as shown in Fig. 8.

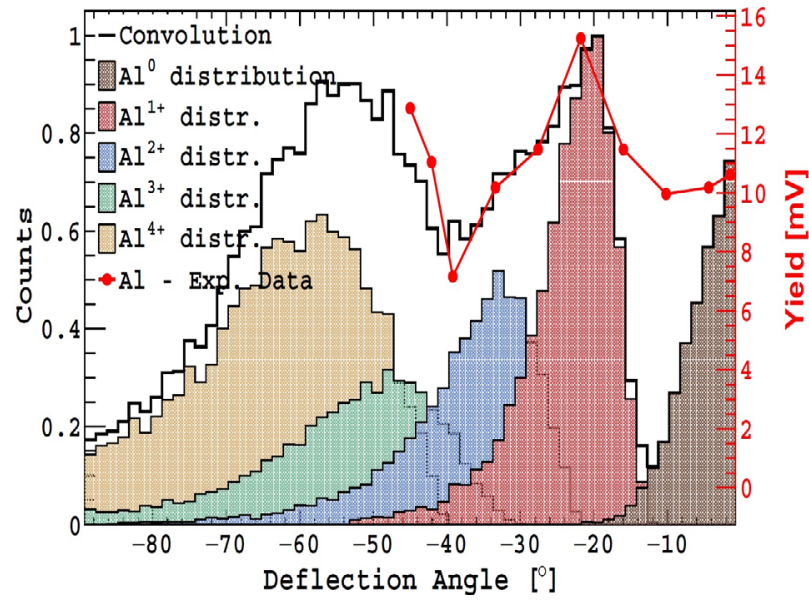

Fig. 8. Comparison of experimental spectrum and convolution of angular distributions of ionic species from $1+$ to $4+$ of aluminium.

From evaluating the number of deflected particles at a certain angle by the magnetic field, for each charge state from $1+$ to $4+$ of $\mathrm{Al}$, the count-angle histogram is obtained. Each count represent the normalized number of particles deflected at a given angle. From the convolution of the simulations from $1+$ to $4+$ charge states of Al-ions, and considering the neutral components $\mathrm{Al}^{0}$ distribution, the ion angular distribution measured and calculated by COMSOL were compared to each other, as shown in Fig. 8. From the excellent agreement between experimental data and simulation results, it was possible to estimate a mean plasma temperature of about $38 \mathrm{eV}$, and an average plasma potential of about $57 \mathrm{~V}$.

\subsection{Transverse Magnetic and Electrical Field}

In this case, two spectrograms were analyzed, that were obtained, with the TPS at LNS in Catania, and with the TPS at PALS in Prague.

The spectrogram at LNS was obtained irradiating with $10^{10} \mathrm{~W} / \mathrm{cm}^{2}$ laser intensity a titanium target. It was achieved using a post ion acceleration from $1 \mathrm{kV}$ up to 30 $\mathrm{kV}$ and Gaf-Chromic films as ions detector [8].

The spectrogram at PALS was obtained irradiating with $10^{16} \mathrm{~W} / \mathrm{cm}^{2}$ laser intensity a deuterated polyethylene and using a Micro Channel Plate coupled to a phosphor screen as ions detector [12].
The overlapping between experimental spectrograms and simulation of the particle trajectories, calculated with COMSOL, allowed to determine the species analyzed for the measurements obtained at LNS shown in Fig. 9a, and to determine the species and energies for the spectrogram obtained at PALS, as shown in Fig. 9b. In the latter case, energy of $3 \mathrm{MeV}$ for charge status was estimated, according to previous papers [13].
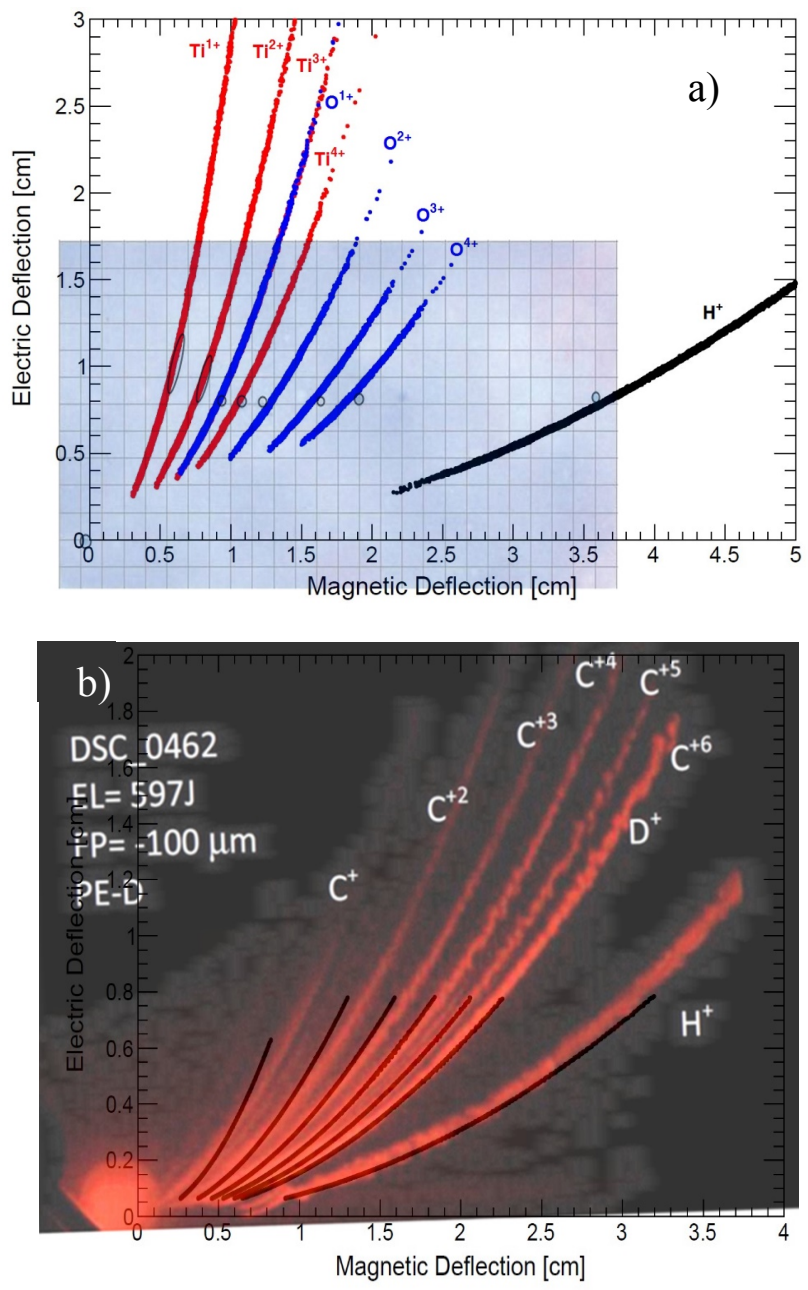

Fig. 9. Comparison between experimental spectrum and simulation, for spectrogram at LNS in Catania (a) and for spectrogram at PALS in Prague (b).

Fig. 9a shows the identifications of parabolas due to protons, titanium and oxygen ions, emitted from the target and post accelerated from $1 \mathrm{keV}$ up to $30 \mathrm{keV}$ per charge state. Due to the maximum charge state of $4+$, it means that the maximum ion energy is $120 \mathrm{keV}$ for oxygen and titanium and $30 \mathrm{keV}$ for protons.

Fig.9b shows the identifications of parabolas due to protons, deuterons and carbon ions emitted form the hot plasma of PALS. In this case the acceleration was of about $3 \mathrm{MeV}$ per charge state, thus the maximum kinetic energy was $18 \mathrm{MeV}$ for Carbon ions and $3 \mathrm{MeV}$ for protons. Deuterium ions are indistinguishable from $\mathrm{C}^{+}+$ ions due to the same mass-to-charge ratio. 


\section{Conclusions}

In this work, we have emphasized the role of simulation programs, like COMSOL Multiphysics, which can be used in the analysis of the dynamics of a nonequilibrium plasma generated by high intensity laser pulses [3]. The simulation code can be used to characterize the plasmas and to study the kinetic energies of the accelerated charged particles.

Indeed, thanks to the present study, it was possible to explain the increase in detected yield and energy observed using magnetic fields with axial symmetry; in this case the simulations carried out with COMSOL Multiphysics have shown that the electrons remain trapped for times of the order of hundreds of nanoseconds; this is sufficient to accelerate ions at higher velocity.

Another result of this study is the analysis of the angular distribution of ions deflected by a magnetic field perpendicular to their motion, which made it possible to estimate the average plasma temperature and potential, both relevant for plasma diagnostics.

Finally, thanks to the study of ions trajectories deflected from magnetic and electrical fields, it was possible to characterize the species that constitute the plasmas, and to evaluate their energy using TPS spectrometers.

All of these studies can result in great benefits to innumerable applications where high intensity laser pulses are used to accelerate ions from solid matter.

This work was supported by the "Research and Mobility" project of Messina University No. 74893496, scientifically coordinated by Professor L. Torrisi.

\section{References}

1. K. Takahaschi, et al., Plasma and Fusion Research, 8, 1206005 (2013)

2. N. Izumi, Y. Sentoku, H. Habara,1, K. Takahashi, et al., Physical Review E. 65(3), 036413 (2002)

3. COMSOL Multiphysics (2017). Actual website: https://www. comsol.it/comsol-multiphysics

4. L. Torrisi, D. Margarone, S. Gammino, L. Andò, Laser and Part. Beam, 25(3), 453-464 (2007)

5. D.C. Carroll, P. Brummitt, D. Neely, F. Lindau, et al., Nucl. Instr. and Meth. in Phys. Res. Sec. A : Acc., Spectr., Detec. and Assoc. Equip., 620(1), 2327 (2010)

6. L. Torrisi and G. Costa, Laser and Part. Beam, 35(2), 202-209 (2017)

7. L. Torrisi, S. Cavallaro, L. Giuffrida, S. Gammino and L. Andò, Rad. Eff. and Def. in Sol. : Inc. Plasma Sci. \& Plasma Tech., 165(6-10), 509-520 (2010)

8. L. Torrisi et al., LNS Activity Report : Ion Sources and Accelerators, 176-179 (2010)

9. M. Passoni, L. Bertagna, and A. Zani, New J. Phys. 12, 045012 (2010)
10. M. Cutroneo, L. Torrisi, et al., Acta Polytechnica, 53(2), 138-141 (2013)

11. L. Torrisi, Rad. Eff. and Def. In Sol., 171(1-2), 3444 (2016)

12. L. Torrisi, M. Cutroneo, et al., EPJ Web of Conferences, 96, 01032 (2015)

13. L. Torrisi et al., LNS Activity Report: Ion Source and Accelerators, 223-226 (2012) 